\title{
Tác động của một số yếu tố kinh tế vĩ mô đến chỉ số giá chứng khoán tại Việt Nam
}

\section{The impact of some macroeconomic factors on stock price Index in Vietnam}

\begin{tabular}{|c|c|}
\hline \multicolumn{2}{|c|}{$\begin{array}{l}{ }^{1} \text { Trường Đại học Ngân hàng Thành phố Hồ Chí Minh, Việt Nam } \\
\text { *Tác giả liên hệ, Email: quynhntn@ buh.edu.vn }\end{array}$} \\
\hline THÔNG TIN & TÓM TẮT \\
\hline $\begin{array}{l}\text { DOI: } 10.46223 / \mathrm{HCMCOUJS} \text {. } \\
\text { econ.vi.14.3.477.2019 }\end{array}$ & $\begin{array}{l}\text { Nghiên cứu nhằm đo lường tác động của } 6 \text { yếu tố kinh tế } \\
\text { vĩ mô bao gồm: giá dầu, chỉ số giá tiêu dùng (đại diện cho lạm } \\
\text { phát), cung tiền } \mathrm{M} 2 \text {, lãi suất, tỷ giá hối đoái và giá vàng đến thị }\end{array}$ \\
\hline Ngày nhận: 17/08/2019 & trường chứng khoán (TTCK) Việt Nam (thông qua chỉ số giá \\
\hline Ngày nhận lại: 05/10/2019 & $\begin{array}{l}\text { chứng khoán VN-Index) trong giai đoạn 2000-2018 bằng mô hình } \\
\text { VECM Kết auả nohiên cứu cho thấv trong dài han lam phát tác }\end{array}$ \\
\hline Duyệt đăng: 07/10/2019 & $\begin{array}{l}\text { động tích cực đến chỉ số VN-Index, lãi suất tác động tiêu cực đến } \\
\text { chỉ số này. Còn trong ngắn hạn, chỉ số VN-Index chủ yếu chịu } \\
\text { tác động bởi chỉ số VN-Index tháng liền trước. Bên cạnh đó, chỉ }\end{array}$ \\
\hline Tù khóa: & $\begin{array}{l}\text { số VN-Index có mối quan hệ cùng chiều với lãi suất, cung tiền, } \\
\text { giá dầu và quan hệ ngược chiều với lạm phát và tỷ giá. Giá vàng }\end{array}$ \\
\hline $\begin{array}{l}\text { chỉ sô giá chứng khoán, } \\
\text { VECM, yếu tố kinh tế vĩ mô }\end{array}$ & $\begin{array}{l}\text { là nhân tố không tác động đáng kể đến chỉ số VN-Index trong cả } \\
\text { ngắn và dài hạn. }\end{array}$ \\
\hline
\end{tabular}

ABSTRACT
This study aims to measure the impact of six macroeconomic
factors including oil price, consumer price index (representing
inflation), M2 money supply, interest rate, exchange rate and gold
price on Vietnamese stock market (via VN-Index stock price
index) in the 2000-2018 period by using VECM model. The
results show a positive correlation between inflation and VN-
Index and a negative correlation between interest rates and the
index in the long run. For short-term periods, VN-Index is mainly
affected by the previous month indexes. It also has a positive
relationship with interest rates, money supply and oil prices and
a negative relationship with inflation and exchange rates. The
effect of gold price on VN-Index is not significant in both short
and long terms.

Keywords:

macroeconomic factors, stock price index, VECM 


\section{Giới thiệu}

TTCK là một trong những nơi đầu tư tài chính phổ biến. Nếu không có TTCK và sự phát triển của thị trường tài chính sẽ không có sự tăng trưởng đáng kể trong nền kinh tế của quốc gia (Hafer \& Hein, 2007). Mặc dù trải qua 18 năm hình thành và phát triển, nhưng TTCK Việt Nam nhìn chung vẫn còn non trẻ và ẩn chứa bên trong nhiều biến động. Do đó, việc phân tích các yếu tố tác động đến TTCK, nhất là các yếu tố vĩ mô tác động đến thị trường này là hết sức hữu ích và cần thiết.

Lý thuyết thị trường hiệu quả do Fama phát triển năm 1970 cho rằng: Giá cả của các chứng khoán trên thị trường tài chính phản ánh đầy đủ mọi thông tin mà nhà đầu tư đã biết (Malkiel \& Fama, 1970). Tuy nhiên, kết quả của nhiều nghiên cứu thực tế trong thời gian gần đây có thể kể đến như nghiên cứu của Akbar, Iqbal, và Noor (2019); Wei, Qin, Li, Zhu, và Wei (2019); González, Nave, và Rubio (2018); Tran (2017), ... đã chứng minh phần lớn thị trường là không hiệu quả, tức là giá cả của các loại chứng khoán chưa thực sự phản ánh đúng thực tế của thị trường do ảnh hưởng của nhiều yếu tố khác.

Cho đến nay đã tồn tại một số lượng các nghiên cứu về tác động của các nhân tố vĩ mô đến TTCK hoặc giá chứng khoán như nghiên cứu của Akbar và cộng sự (2019); Singhal, Choudhary, và Biswal (2019); González và cộng sự (2018); ... Tại Việt Nam, đã có một vài nghiên cứu phân tích chủ đề này như Nguyen và Nguyen (2013); Truong (2014); Dinh và Nguyen (2008). Mặc dù vậy, các nghiên cứu này chủ yếu tập trung vào việc đo lường ảnh hưởng của các yếu tố vĩ mô đến giá của cổ phiếu, hoặc nếu nghiên cứu về chỉ số giá chứng khoán VNIndex thì chỉ dừng lại một số chỉ tiêu cơ bản như lạm phát, tỷ giá, cung tiền, giá vàng mà chưa có nghiên cứu tổng thể về các nhân tố vĩ mô tác động đến chỉ số giá chứng khoán VN-Index tại nước ta.

Với nghiên cứu này, các tác giả kiểm tra tác động của 6 yếu tố kinh tế vĩ mô đến TTCK Việt Nam (thông qua chỉ số giá thị trường chứng khoán VN-Index) trong giai đoạn từ tháng $12 / 2000$ đến đến tháng $12 / 2018$. Kết quả của nghiên cứu là cơ sở cho nhà đầu tư có thêm thông tin để đưa ra quyết định phù hợp đồng thời củng cố thêm bằng chứng thực nghiệm để các nhà làm chính sách đưa ra các giải pháp phát triển TTCK Việt Nam. Phần tiếp theo của bài viết sẽ trình bày cơ sở lý thuyết và các yếu tố vĩ mô tác động đến TTCK, phần 3 là phương pháp, mô hình và dữ liệu nghiên cứu, phần 4 là thảo luận kết quả nghiên cứu và phần 5 là kết luận và khuyến nghị một số chính sách.

\section{Cơ sở lý thuyết}

\subsection{Chỉ số giá chúng khoán}

Chỉ số giá chứng khoán là chỉ báo giá cổ phiếu phản ánh xu hướng phát triển của thị trường cổ phiếu, thể hiện xu hướng thay đổi của giá cổ phiếu và tình hình giao dịch trên thị trường. Đơn giản, chỉ số giá chứng khoán là giá bình quân cổ phiếu tại một ngày nhất định so

với ngày gốc (Bui, 2013). Tại Việt Nam, chỉ số VN-Index là chỉ số giá chứng khoán của sàn 
giao dịch chứng khoán thành phố Hồ Chí Minh (HOSE), thể hiện xu hướng biến động giá của toàn bộ cổ phiếu niêm yết trên HOSE.

Chỉ số VN-Index thể hiện mức biến động giá, được tính theo phương pháp bình quân gia quyền của toàn bộ các cổ phiếu trong rổ tính toán so với phiên giao dịch gần nhất hoặc so với ngày gốc và được tính theo điểm. Về nguyên tắc, $\mathrm{VN}$-Index được tính bằng phương pháp Passher theo công thức:

$$
\text { VnIndex }=\frac{\sum_{\mathrm{i}}\left(\mathrm{p}_{\mathrm{it}} \times \mathrm{q}_{\mathrm{it}}\right)}{\sum_{\mathrm{i}}\left(\mathrm{p}_{\mathrm{it}} \times \mathrm{q}_{\mathrm{i} 0}\right)} \times 100
$$

Trong đó:

VnIndex: Là chỉ số giá VN-Index được tính theo phương pháp Paasche.

$\mathrm{p}_{\mathrm{it}}$ : Là giá cổ phiếu $\mathrm{i}$ thời kỳ $\mathrm{t}$.

$\mathrm{p}_{\mathrm{i} 0}$ : Là giá cổ phiếu i thời kỳ gốc.

$\mathrm{q}_{\mathrm{it}}$ : Là khối lượng (quyền số) thời điểm tính toán ( $\mathrm{t}$ ) hoặc cơ cấu của khối lượng thời điểm tính toán.

i: Là cổ phiếu i tham gia tính chỉ số giá.

Trong đó, cách chọn rổ đại diện (chọn các cổ phiếu i để tham gia tính toán) của thị trường bao gồm các cổ phiếu tiêu biểu và đại diện được cho tổng thể thị trường. Do đó, các cổ phiếu trong rổ đại diện thường xuyên được thay đổi do phải thay đổi những cổ phiếu không còn tiêu biểu bằng các cổ phiếu tiêu biểu hơn. Ba tiêu thức quan trọng để xác định sự tiêu biểu của cổ phiếu là (i) số lượng cổ phiếu niêm yết, giá trị niêm yết và (iii) tỷ lệ giao dịch chứng khoán đó trên thị trường.

\subsection{Các yếu tố vĩ mô tác động đến thị truờng chứng khoán}

Theo khảo lược của các tác giả, cho đến nay, khi nghiên cứu về tác động của các yếu tố vĩ mô đến TTCK có hai hướng nghiên cứu chính. Một là, các nghiên cứu về tác động của các yếu tố kinh tế vĩ mô đến chỉ số giá chứng khoán - giá cổ phiếu trên TTCK Abdalla và Murinde (1997); Adam và Tweneboah (2008); Nguyen và Nguyen (2013); ... Hai là, các nghiên cứu về tác động của các yếu tố đến biến động lợi nhuận chứng khoán của các công ty như nghiên cứu của Su, Fang, và Yin (2019); Schwert (1989). Bài viết này tập trung theo hướng nghiên cứu thứ nhất là xác định các yếu tố vĩ mô đến chỉ số chứng khoán.

\section{Lạm phát}

Lạm phát là hiện tượng xảy ra khi mức giá chung trong nền kinh tế tăng kéo dài trong một khoảng thời gian nhất định (Le \& Dang, 2017).

Kết quả nghiên cứu của Adam và Tweneboah (2008) tìm thấy mối quan hệ tương quan cùng chiều giữa lạm phát và và chỉ số giá TTCK. Kết quả này hàm ý rằng thị trường phân bổ hiệu quả các nguồn lực bằng cách điều chỉnh tăng theo mức giá tăng chung trong dài hạn. Tuy vậy, phần lớn các nghiên cứu khác kết luận rằng lạm phát tác động ngược chiều với TTCK. Cụ 
thể có thể kể đến nghiên cứu của Subhani, Gul, và Osman (2010); Geetha, Mohidin, Chandran, và Chong (2011); Mousa, Safi, Hasoneh, và Mohammad (2012). Thật vậy, khi lạm phát tăng cao, tiền mất giá, người dân có xu hướng nắm giữ các tài sản thực như vàng, bất động sản,... thay vì các tài sản có tính thanh khoản cao như tiền mặt, tiền gửi ngân hàng, hoặc các tài sản tài chính như trái phiếu, cổ phiếu điều này khiến cho một lượng vốn nhàn rỗi đáng kể của xã hội nằm im dưới dạng tài sản chết. Bên cạnh đó, đối với các doanh nghiệp sản xuất kinh doanh, lạm phát tăng làm cho chi phí của doanh nghiệp bị đẩy lên, dẫn đến giá hàng hóa tăng, khiến lượng cầu sụt giảm, dẫn đến doanh thu của doanh nghiệp giảm. Doanh nghiệp không còn hấp dẫn đối với nhà đầu tư trên TTCK. "Tâm lý bầy đàn" xuất hiện, kéo theo việc rút vốn ồ ạt trên TTCK. Có thể thấy lạm phát quá cao tác động xấu đến nền kinh tế nói chung và TTCK nói riêng.

\section{Cung tiền}

Mức cung tiền tệ là số lượng tiền tệ thực tế trong lưu thông, thường được xác định trong một thời kỳ nhất định. Lượng tiền tệ thực tế trong lưu thông không chỉ bao gồm tiền mặt, tiền gửi thanh toán trong ngân hàng mà còn gồm nhiều tài sản khác có tính lỏng cao, dễ dàng chuyển sang tiền mặt và được chấp nhận rộng rãi trong thanh toán, giao dịch. Mối quan hệ giữa cung tiền và TTCK là mối quan hệ cùng chiều được thể hiện thông qua CSTT. Friedman và Schwartz (1965) đưa ra lời giải thích đầu tiên về mối quan hệ này, theo đó một sự gia tăng cung tiền sẽ làm gia tăng thanh khoản và tín dụng cho nhà đầu tư cổ phiếu dẫn đến giá chứng khoán tăng.

Nguyen và Nguyen (2013) cũng đã phân tích và đồng quan điểm khi cho rằng cung tiền có quan hệ cùng chiều với TTCK. Khi lượng cung tiền tăng, thanh khoản vượt trội sẽ ảnh hưởng đến TTCK khá mạnh do tác động của CSTT. Trong trường hợp CSTT mở rộng, lãi suất của nền kinh tế giảm, làm lãi suất chiết khấu của chứng khoán giảm qua đó làm tăng giá kỳ vọng và tăng thu nhập. Trong trường hợp CSTT thắt chặt, lãi suất tăng cao làm tăng lãi suất chiết khấu của mô hình định giá, làm cho các chứng khoán thu nhập cố định trở thành hấp dẫn hơn, làm giảm thanh khoản vào cổ phiếu, giảm xu hướng vay mượn để đầu tư vào chứng khoán và cuối cùng làm ảnh hưởng đến lợi nhuận doanh nghiệp, tác động làm giảm giá chứng khoán. Nghiên cứu thực nghiệm của Rahman, Sidek, và Tafri (2009); Rozeff (1974) khẳng định CSTT có tác động đáng kể đến tăng trưởng của lợi nhuận chứng khoán. Maysami và Koh (2000) bổ sung thêm một mối quan hệ tích cực giữa cung tiền và chỉ số SGX (Chỉ số TTCK Singapore).

\section{Tỷ giá}

Theo Cecchetti, Schoenholtz, và Fackler (2006) tỷ giá hay tỷ giá hối đoái là tỷ lệ trao đổi từ tiền của quốc gia này sang đồng tiền của quốc gia khác. Tỷ giá hối đoái là một biến số rất quan trọng, tác động đến sự cân bằng của cán cân thương mại và cán cân thanh toán, do đó tác động đến sản lượng, việc làm cũng như sự cân bằng của nền kinh tế nói chung. Ngoài ra tỷ giá còn ảnh hưởng trực tiếp đến các doanh nghiệp trong nền kinh tế.

Tại Việt Nam, kết quả nghiên cứu của Dinh và Nguyen (2008) đã chỉ ra tỷ giá là yếu tố gây bất lợi cho TTCK. Khi tỷ giá (VND/USD) tăng, đồng nghĩa với việc USD tăng giá và VND 
mất giá. Khi đó, 1 USD sẽ đổi được nhiều VND hơn và sẽ có một khoản lời gia tăng từ việc chuyển đổi từ USD sang VND. Khi đó, nhà đầu tư có thể thu được lợi nhuận nhiều hơn so với đầu tư vào TTCK. Tuy nhiên, kết quả một số nghiên cứu khác lại cho thấy tác động tích cực giữa tỷ giá và chỉ số giá TTCK của quốc gia này nhưng lại tác động tiêu cực với quốc gia khác. Theo Ho và Huang (2015), tỷ giá tác động đến chỉ số giá chứng khoán của Brazil, Ân Độ và Nga nhưng không ảnh hưởng đến TTCK Trung Quốc. Abdalla và Murinde (1997) tìm thấy mối quan hệ nhân quả giữa tỷ giá và TTCK tại Ấn Độ, Nam Hàn Quốc và Pakistan nhưng Philippines thì không. Bằng mô hình VAR, Rjoub (2012) đã chứng minh trong dài hạn, tỷ giá tác động tiêu cực đến TTCK Mỹ và tích cực đến TTCK Thổ Nhĩ Kỳ giai đoạn 2001-2009.

Như vậy, có thể thấy mối quan hệ giữa tỷ giá và chỉ số giá chứng khoán vẫn là một câu hỏi cần lời giải đáp, các nghiên cứu thực nghiệm đã chỉ ra tác động giữa tỷ giá đối với TTCK ở từng thị trường và hoàn cảnh khác nhau có thể cho ra kết quả khác nhau.

\section{Lãi suất}

Lãi suất là chi phí mà người đi vay phải trả để có được cơ hội sử dụng vốn (Devereux \& Yetman, 2002), được xác định bởi nguồn cung và nhu cầu sử dụng vốn. Khi nhu cầu về vốn tăng, lãi suất sẽ tăng và ngược lại. Với quan điểm của doanh nghiệp, tín dụng để tài trợ vốn lưu động hoặc chi tiêu, sẽ gia tăng chi phí nợ. Điều này tác động làm giảm lợi nhuận của doanh nghiệp và cổ tức của cổ đông. Do đó, giá cổ phiếu có thể giảm. Mặt khác, lãi suất cao hơn làm giảm giá trị của thu nhập cổ tức trong tương lai, điều này sẽ khiến nhà đầu tư ngại đầu tư vào cổ phiếu.

Nhiều nghiên cứu đã chỉ ra mối quan hệ giữa lãi suất và giá cổ phiếu thường là quan hệ ngược chiều. Alam và Uddin (2009) kết luận hầu hết giá chứng khoán của các nước chịu tác động tiêu cực của lãi suất, điều này cũng từng được Adam và Tweneboah (2008) chứng minh.

\section{Giá vàng}

Vàng là phương tiện cất trữ giá trị. Khác với các tài sản khác, vàng có tiềm năng là tính thanh khoản cao. Sự biến động của giá vàng ảnh hưởng đến hầu hết các nền kinh tế trong đó có TTCK. Cơ sở để giải thích cho mối tương quan này là khi giá vàng tăng, nhà đầu tư sẽ rút vốn để đầu tư vào thị trường vàng thay vì đầu tư vào cổ phiếu do suất sinh lợi trên thị trường vàng cao hơn. Do đó, cầu về cổ phiếu sẽ giảm, làm giảm giá của cổ phiếu.

Theo Truong (2014) sự biến động của giá vàng có tương quan nghịch với tỷ suất sinh lời của các cổ phiếu. Một cách định lượng, khi giá vàng tăng hoặc giảm $1 \%$ thì tỷ suất sinh lời của các cổ phiếu sẽ giảm hoặc tăng $0,72 \%$. Bằng mô hình VAR, Akbar và cộng sự (2019), cũng chứng minh giá vàng và giá chứng khoán có tác động qua lại lẫn nhau và đó là tác động ngược chiều.

\section{Giá dầu}

Dầu là nguồn năng lượng quan trọng, là nhiên liệu vận chuyển thiết yếu không thể thay thế trong nhiều ngành công nghiệp. Ngoài ra yếu tố này còn là mặt hàng kinh doanh quan trọng 
của thế giới. Giá dầu tăng ảnh hưởng đến các biến số kinh tế vĩ mô như chi phí sản xuất, quyết định của nhà đầu tư, các biến số kinh tế vĩ mô như lạm phát, thu nhập quốc dân, ... Vì vậy, sự thay đổi giá dầu được kỳ vọng sẽ có những ảnh hưởng nhất định đến TTCK.

Mối quan hệ giữa giá dầu và giá chứng khoán có thể tích cực hoặc tiêu cực. Thứ nhất, vì dầu là nguồn năng lượng đầu vào chính yếu của hầu hết các công ty, khi giá dầu tăng làm đội chi phí lên cao, từ đó làm giảm lợi nhuận công ty. Thứ hai, giá dầu tăng có thể làm tăng lạm phát kỳ vọng và lãi suất từ đó làm giảm lợi nhuận từ dòng cổ tức trong tương lai (Smyth \& Narayan, 2018). Mặt khác, giá dầu cao hơn có thể phản ánh hiệu quả kinh doanh tốt hơn đồng thời giá dầu tăng phản ánh thị trường đang tăng trưởng và mức độ tự tin kinh doanh cao (Hamilton, 2008). Do đó, giá dầu cũng có tác động tích cực đến TTCK.

\section{Phương pháp nghiên cứu}

\subsection{Mô hình nghiên cứu}

Dựa trên những nghiên cứu thực nghiệm trước đây, mô hình tự hồi quy vector (VAR), mô hình hiệu chỉnh sai số vectơ (VECM) được đề xuất để nghiên cứu tác động của các yếu tố vĩ mô đến chỉ số giá chứng khoán. Tuy nhiên, với dạng dữ liệu nghiên cứu là dữ liệu chuỗi thời gian, và bằng chứng có hiện tượng đồng liên kết đối với dữ liệu trong bài nghiên cứu, các tác giả sử dụng mô hình hiệu chỉnh sai số vectơ (VECM) theo phương pháp Johansen để ước lượng. Mô hình VECM bằng phương pháp Johansen có dạng:

$$
\Delta \mathrm{Y}_{\mathrm{t}}=\Gamma_{1} \Delta \mathrm{Y}_{\mathrm{t}-1}+\Gamma_{2} \Delta \mathrm{Y}_{\mathrm{t}-2}+\cdots+\Gamma_{\mathrm{k}-1} \Delta \mathrm{Y}_{\mathrm{t}-(\mathrm{k}-1)}+\Pi \mathrm{Y}_{\mathrm{t}-1}+\mathrm{u}_{\mathrm{t}}
$$

Trong đó, $\Gamma_{\mathrm{i}}=\left(\sum_{\mathrm{j}=1}^{\mathrm{k}} \beta_{1}\right)-\mathrm{I}_{\mathrm{g}}$ và $\Pi=\left(\sum_{\mathrm{i}=1}^{\mathrm{k}} \beta_{1}\right)-\mathrm{I}_{\mathrm{g}}$

Mô hình này gồm $\mathrm{g}$ biến số dạng sai phân ở vế trái và $(\mathrm{k}-1)$ bậc trễ của biến độc lập ở dạng sai phân ở vế phải, mỗi biến có $\Gamma$ ma trận hệ số. Ma trận $\Pi(\mathrm{g} \times \mathrm{g})$ là ma trận hệ số phản ánh quan hệ dài hạn giữa các biến số tại mức cân bằng, tất cả $\Delta \mathrm{Y}_{\mathrm{t}-\mathrm{i}}=0$, sai số $\mathrm{u}_{\mathrm{t}}=0$ khi đó $\Pi \mathrm{Y}_{\mathrm{t}-1}=0$. Ma trận $\Pi$ là tích phân của hai ma trận $\alpha(\mathrm{g} \times \mathrm{r})$ và $\beta^{1}(\mathrm{~g} \times \mathrm{r})$ với $\mathrm{r}$ là số lượng vectơ đồng liên kết cũng đồng thời là bậc của ma trận ח:

$$
\Pi=\alpha \cdot \beta^{1}
$$

Trong đó: ma trận $\beta^{\prime}$ là ma trận vectơ đồng liên kết, phản ánh quan hệ dài hạn giữa các biến số; là hệ số của vectơ đồng liên kết trong VECM. Do đó, VECM chứa mô hình hồi quy đồng liên kết giúp phân tích tác động dài hạn và phần còn lại của VECM cho ta biết tác động trong ngắn hạn của các biến độc lập đến biến phụ thuộc.

Trong bài nghiên cứu này, tác giả chủ yếu dựa theo mô hình nghiên cứu của (Adam \& Tweneboah, 2008) với các biến lạm phát (CPI), lãi suất (IR), tỷ giá $(\mathrm{EX})$ và bổ sung thêm các biến: cung tiền (M2) theo (Rahman et al., 2009), giá dầu (OP) theo (Mokni \& Youssef, 2019), giá vàng (GP) theo (Singhal et al., 2019). Do các biến có sự biến động lớn nên trước khi chạy mô hình, các tác giả lấy logarit các biến để tăng tính ổn định. Như vậy mô hình nghiên cứu được đề xuất như sau:

$$
\text { LVNI = F(LOP, LCPI, LM2, LIR, LEX, LGP) }
$$


Các biến và dầu kỳ vọng trong mô hình nghiên cứu được thể hiện như sau (Bảng 1):

\section{Bảng 1}

Miêu tả các biến số trong mô hình

\begin{tabular}{|c|c|c|c|c|}
\hline Tên biến & $\begin{array}{c}\text { Ký } \\
\text { hiệu }\end{array}$ & Đơn vị & Các nghiên cứu & $\begin{array}{c}\text { Kỳ } \\
\text { vọng }\end{array}$ \\
\hline $\begin{array}{l}\text { Chỉ số giá } \\
\text { chứng khoán } \\
\text { VN-Index }\end{array}$ & VNI & Điểm & $\begin{array}{l}\text { (Nguyen \& Nguyen, 2013; Truong, } \\
\text { 2014) }\end{array}$ & \\
\hline Giá dầu & $\mathrm{OP}$ & USD/thùng & $\begin{array}{l}\text { (Hamilton, 2008; Mokni \& Youssef, } \\
\text { 2019; Smyth \& Narayan, } 2018 \text { ) }\end{array}$ & $(+/-)$ \\
\hline Lạm phát & CPI & $\%$ & $\begin{array}{l}\text { (Adam \& Tweneboah, 2008; Geetha et } \\
\text { al., 2011; Mousa et al., 2012; Subhani et } \\
\text { al., 2010) }\end{array}$ & $(+/-)$ \\
\hline Cung tiền & M2 & Tỷ đồng & (Rahman et al., 2009; Rozeff, 1974) & $(+)$ \\
\hline Lãi suất & IR & \%/năm & $\begin{array}{l}\text { (Adam \& Tweneboah, 2008; Alam \& } \\
\text { Uddin, 2009) }\end{array}$ & $(-)$ \\
\hline Tỷ giá hối đoái & EX & VND/USD & $\begin{array}{l}\text { (Abdalla \& Murinde, 1997; Adam \& } \\
\text { Tweneboah, 2008; Ho \& Huang, 2015) }\end{array}$ & $(+/-)$ \\
\hline Giá vàng & GP & VND/lượng & $\begin{array}{l}\text { ( Akbar et al., 2019; Singhal et al., 2019; } \\
\text { Truong, 2014) }\end{array}$ & $(-)$ \\
\hline
\end{tabular}

Nguồn: Thu thập và tổng hợp của tác giả

\subsection{Dũ liệu nghiên cứu}

Dữ liệu nghiên cứu được thu thập là dữ liệu chuỗi thời gian theo tháng trong giai đoạn 12/2000 đến 12/2018. Cụ thể dữ liệu về chỉ số giá VN-Index thu thập từ website Tổng công ty cổ phần chứng khoán VNDirect, dữ liệu giá dầu được lấy từ FRED Economy Data, giá vàng lấy từ hội đồng vàng thế giới (The World Gold Council), dữ liệu của các yếu tố khác như lạm phát, lãi suất, tỷ giá hối đoái, cung tiền được thu thập từ Quỹ tiền tệ Quốc tế (IMF). Kết quả thống kê mô tả các biến sử dụng trong nghiên cứu được thể hiện trong Bảng 2.

\section{Bảng 2}

Thống kê mô tả các biến trong mô hình 


\begin{tabular}{|c|c|c|c|c|c|}
\hline & Trung bình & Tối đa & Tối thiểu & Độ lệch chuẩn & Số quan sát \\
\hline CPI & 100.87 & 163.58 & 47.64 & 40.91 & 217 \\
\hline EX & $18,493.93$ & $22,783.04$ & $14,099.00$ & $2,840.52$ & 217 \\
\hline GP & $18,749,506$ & $36,902,806$ & $3,791,467$ & $10,737,491$ & 217 \\
\hline IR & 7.50 & 17.16 & 4.24 & 2.93 & 217 \\
\hline M2 & $2,775,763$ & $8,760,391$ & 196,994 & $2,539,389$ & 217 \\
\hline OP & 66.38 & 132.72 & 18.71 & 30.4 & 217 \\
\hline VNI & 499.57 & $1,174.46$ & 136.21 & 248.52 & 217 \\
\hline
\end{tabular}

Nguồn: Kết quả thống kê bằng Eview 10.0

\section{Kết quả nghiên cứu và thảo luận}

\subsection{Kiểm định tính dùng}

Các tác giả sử dụng một phương pháp khá phổ biến đó là kiểm định nghiệm đơn vị (Unit Root Test) để kiểm định tính dừng của chuỗi dữ liệu.

\section{Bảng 3}

Kết quả kiểm định nghiệm đơn vị

\begin{tabular}{|c|c|c|c|c|}
\hline \multirow{2}{*}{ Biến } & \multicolumn{4}{|c|}{ Giá trị p-value } \\
\cline { 2 - 5 } & Chuỗi gốc & Sai phân bậc 1 & Chuỗi gốc & Sai phân bậc 1 \\
\hline LVNI & 0.1588 & 0.0000 & 0.3419 & 0.0000 \\
\hline LOP & 0.6371 & 0.0000 & 0.7327 & 0.0000 \\
\hline LCPI & 0.9778 & 0.0000 & 0.9679 & 0.0000 \\
\hline LM2 & 0.9994 & 0.0000 & 0.9981 & 0.0000 \\
\hline LIR & 0.6284 & 0.0000 & 0.4638 & 0.0000 \\
\hline LEX & 0.8581 & 0.0000 & 0.4638 & 0.0000 \\
\hline LGP & 0.9914 & 0.0000 & 0.9896 & 0.0000 \\
\hline
\end{tabular}

Nguồn: Kết quả kiểm định bằng Eview 10.0

Nghiên cứu thực hiện kiểm định nghiệm đơn vị bằng 2 kiểm định: Kiểm định $\mathrm{ADF}$ bắt nguồn từ kiểm định Dickey và Fuller (DF) do Dickey và Fuller (1979) đưa ra và mở rộng thành $\mathrm{ADF}, \mathrm{Kiể}$ định $\mathrm{PP}$ do Phillips và Perron (1988) phát triển. 
Bảng 3 cho thấy trong cả kiểm định $\mathrm{ADF}$ và $\mathrm{PP}, \mathrm{p}$-value $>10 \%$ ở tất cả các biến chuỗi gốc và $p$-value $<1 \%$ ở dạng sai phân bậc 1 . Kết luận, tất cả các biến đều không dừng ở dạng gốc nhưng dừng ở sai phân bậc 1 .

\subsection{Lụa chọn độ trễ}

Bảng 4 cho thấy kết quả lựa chọn độ trễ tối ưu theo 5 tiêu chuẩn khác nhau. Phần mềm Eview 10.0 đã giúp chỉ ra độ trễ tối ưu ở mỗi tiêu chuẩn bằng cách đánh dấu sao $(*)$. Bài nghiên cứu lựa chọn biến trễ theo kết quả phù hợp với nhiều tiêu chuẩn nhất. Theo đó, các tiêu chuẩn FPE, AIC và HQ cùng ra kết quả số biến trễ tối đa là 1 . Như vậy, độ trễ được lựa chọn để chạy mô hình hồi quy VECM là $\mathrm{k}=1$, tức là giá trị của các biến hiện tại sẽ chịu tác động của giá trị của các biến trễ theo tháng là một tháng trước đó.

\section{Bảng 4}

Kết quả lựa chọn biến trễ

\begin{tabular}{ccccccc}
\hline \hline Lag & LogL & LR & FPE & AIC & SC & HQ \\
\hline \hline & & & & & & \\
0 & -389.0211 & NA & $1.06 \mathrm{e}-07$ & 3.807896 & $3.920217 *$ & 3.853313 \\
1 & -262.8063 & 242.7209 & $5.06 \mathrm{e}-08^{*}$ & $3.065445^{*}$ & 3.964013 & $3.428780^{*}$ \\
2 & -231.9008 & 57.35352 & $6.03 \mathrm{e}-08$ & 3.239430 & 4.924245 & 3.920683 \\
3 & -191.6247 & 72.03214 & $6.58 \mathrm{e}-08$ & 3.323315 & 5.794377 & 4.322485 \\
4 & -152.1962 & 67.86251 & $7.27 \mathrm{e}-08$ & 3.415348 & 6.672657 & 4.732437 \\
5 & -93.71551 & 96.71812 & $6.72 \mathrm{e}-08$ & 3.324188 & 7.367743 & 4.959194 \\
6 & -44.68088 & $77.79532 *$ & $6.83 \mathrm{e}-08$ & 3.323855 & 8.153657 & 5.276779 \\
7 & -17.34587 & 41.52820 & $8.63 \mathrm{e}-08$ & 3.532172 & 9.148221 & 5.803014 \\
8 & 23.91756 & 59.91132 & $9.61 \mathrm{e}-08$ & 3.606562 & 10.00886 & 6.195322 \\
\hline \hline
\end{tabular}

Nguồn: Kết quả kiểm định bằng Eview 10.0

\subsection{Kiểm định đồng liên kết}

Kết quả kiểm định Trace ở Bảng 5 cho thấy, với giả thuyết $\mathrm{H}_{0}$ là $\mathrm{r} \leq 1$, trị thống kê kiểm định Trace là 217.4568 lớn hơn giá trị bác bỏ tại mức ý nghĩa $5 \%$ là 111.7805 , giả thuyết $\mathrm{H}_{0}$ bị bác bỏ tại mức $5 \%$. Tương tự ta bác bỏ giả thuyết $\mathrm{H}_{0}$ là $\mathrm{r} \leq 2$ và $\mathrm{r} \leq 3$ tại mức ý nghĩa $5 \%$. Với giả thuyết $\mathrm{H}_{0}$ là $\mathrm{r} \leq 4$, trong khi giá trị bác bỏ tại mức ý nghĩa $5 \%$ là 40.17493 , giá trị kiểm định thống kê Trace chỉ đạt 39.83304, giả thuyết $\mathrm{H}_{0}$ không thể bị bác bỏ. Tương tự ta chấp nhận giả thuyết $\mathrm{H}_{0}$ tại giá trị $\mathrm{r} \leq 5, \mathrm{r} \leq 6, \mathrm{r} \leq 7$. Như vậy, kiểm định Trace cho thấy có ít nhất 3 đồng liên kết được tìm thấy. 


\section{Bảng 5}

Kết quả kiểm định đồng liên kết Johansen

\begin{tabular}{|c|c|c|c|c|c|c|c|}
\hline \multicolumn{3}{|c|}{ Kiểm định Trace } & \multicolumn{3}{c|}{ Kiểm định Max-Eigen } \\
\hline $\mathbf{H}_{\mathbf{0}}$ & $\mathbf{H}_{\mathbf{1}}$ & $\begin{array}{c}\text { Thống kê } \\
\text { Trace }\end{array}$ & $\begin{array}{c}\text { Giá trị bác } \\
\text { bỏ tại mức } \\
\text { ý nghĩa 5\% }\end{array}$ & $\mathbf{H}_{\mathbf{0}}$ & $\mathbf{H}_{\mathbf{1}}$ & $\begin{array}{c}\text { Thống kê } \\
\text { Max-Eigen }\end{array}$ & $\begin{array}{c}\text { Giá trị bác } \\
\text { bỏ tại mức ý } \\
\text { nghĩa 5\% }\end{array}$ \\
\hline $\mathrm{r} \leq 1$ & $\mathrm{r}>1$ & 217.4568 & 111.7805 & $\mathrm{r}=0$ & $\mathrm{r}=1$ & 110.7858 & 42.77219 \\
\hline $\mathrm{r} \leq 2$ & $\mathrm{r}>2$ & 106.6710 & 83.93712 & $\mathrm{r}=1$ & $\mathrm{r}=2$ & 46.27066 & 36.63019 \\
\hline $\mathrm{r} \leq 3$ & $\mathrm{r}>3$ & 60.40031 & 60.06141 & $\mathrm{r}=2$ & $\mathrm{r}=3$ & 20.56728 & 30.43961 \\
\hline $\mathrm{r} \leq 4$ & $\mathrm{r}>4$ & 39.83304 & 40.17493 & $\mathrm{r}=3$ & $\mathrm{r}=4$ & 18.39886 & 24.15921 \\
\hline $\mathrm{r} \leq 5$ & $\mathrm{r}>5$ & 21.43418 & 24.27596 & $\mathrm{r}=4$ & $\mathrm{r}=5$ & 11.65221 & 17.79730 \\
\hline $\mathrm{r} \leq 6$ & $\mathrm{r}>6$ & 9.781969 & 12.32090 & $\mathrm{r}=5$ & $\mathrm{r}=6$ & 9.577233 & 11.22480 \\
\hline $\mathrm{r} \leq 7$ & $\mathrm{r}>7$ & 0.204736 & 4.129906 & $\mathrm{r}=6$ & $\mathrm{r}=7$ & 0.204736 & 4.129906 \\
\hline
\end{tabular}

Nguồn: Kết quả kiểm định Johansen bằng Eview 10.0

Kiểm định Max-Eigen cũng cho kết quả tương tự, giả thuyết $\mathrm{H}_{0}$ là $\mathrm{r}=0, \mathrm{r}=1$ và $\mathrm{r}=2$ bị bác bỏ tại mức ý nghĩa 5\%, nhưng các giả thuyết $\mathrm{H}_{0}$ là $\mathrm{r}=3, \mathrm{r}=4, \mathrm{r}=5, \mathrm{r}=6$ không bị bác bỏ. Như vậy, kiểm định Max-Eigen cũng cho thấy có 3 vectơ đồng liên kết được tìm thấy.

\subsection{Phân rã phương sai}

Dựa vào kết quả phân rã phương sai (Bảng 7) có thể thấy chỉ số VN-Index chịu tác động rất lớn từ các cú sốc của chính nó tạo ra và kéo dài nhiều tháng sau đó, đến tháng thứ 10 mức độ tự giải thích của LVNI vẫn chiếm đến gần $90.38 \%$ (Bảng 6). Cú sốc giá dầu ở 2 tháng đầu đóng góp rất nhỏ trong việc giải thích biến động của chỉ số VN-Index, nhưng sau đó tăng mạnh ở tháng thứ 3 và chiếm khoảng 3.4\% ở tháng thứ 10 . Trong khi đó, vai trò của cú sốc lạm phát và cung tiền cũng tăng dần và đạt cao nhất ở tháng thứ 10 . Lãi suất, tỷ giá và giá vàng có ảnh hưởng rất nhỏ đến chỉ số VN-Index.

\section{Bảng 6}

Phân rã phương sai

\begin{tabular}{ccccccccc} 
Period & S.E. & D(LVNI) & D(LOP) & D(LCPI) & D(LM2) & D(LIR) & D(LEX) & D(LGP) \\
\hline \hline 1 & 0.089241 & 100.0000 & 0.000000 & 0.000000 & 0.000000 & 0.000000 & 0.000000 & 0.000000 \\
2 & 0.098092 & 95.12043 & 0.424582 & 1.625101 & 2.058717 & 0.013469 & 0.025281 & 0.732422 \\
3 & 0.100249 & 91.07793 & 3.074882 & 1.987763 & 2.314841 & 0.163856 & 0.498672 & 0.882055 \\
4 & 0.100612 & 90.42314 & 3.423746 & 1.986471 & 2.337213 & 0.237474 & 0.663236 & 0.928720 \\
5 & 0.100666 & 90.40740 & 3.421174 & 1.984495 & 2.339601 & 0.237271 & 0.670707 & 0.939349 \\
6 & 0.100691 & 90.39761 & 3.419445 & 1.983505 & 2.348432 & 0.237984 & 0.670370 & 0.942658
\end{tabular}




\begin{tabular}{ccccccccc} 
Period & S.E. & D(LVNI) & D(LOP) & D(LCPI) & D(LM2) & D(LIR) & D(LEX) & D(LGP) \\
\hline \hline 7 & 0.100698 & 90.38769 & 3.419678 & 1.983851 & 2.357006 & 0.238336 & 0.670316 & 0.943118 \\
8 & 0.100701 & 90.38286 & 3.419502 & 1.983769 & 2.361150 & 0.238527 & 0.670458 & 0.943739 \\
9 & 0.100702 & 90.38039 & 3.419608 & 1.983777 & 2.363366 & 0.238642 & 0.670476 & 0.943745 \\
10 & 0.100703 & 90.37860 & 3.419714 & 1.983829 & 2.364971 & 0.238700 & 0.670462 & 0.943725 \\
\hline \hline
\end{tabular}

Cholesky Ordering: D(LVNI) D(LOP) D(LCPI) D(LM2) D(LIR) D(LEX) D(LGP)

Nguồn: Kết quả kiểm định bằng Eview 10.0

\subsection{Kết quả uớc lự̣ng mô hình VECM}

Phương trình đồng liên kết thể hiện tác động dài hạn của các biến độc lập đến VNI được thể hiện qua phương trình:

$$
\begin{aligned}
& \mathrm{LVNI}=0.093832 \mathrm{LOP}+4.642844 \mathrm{LCPI}-1.801236 \mathrm{LIR}-1.043372 \mathrm{LM} 2 \\
& {\left[\begin{array}{lll}
-0.15693 & {[-2.18959] \quad[2.88068]} & {[1.57378]}
\end{array}\right.}
\end{aligned}
$$

Kết quả ước lượng mô hình VECM mô tả tác động ngắn hạn của các biến độc lập đến LVNI thể hiện qua phương trình:

$$
\begin{aligned}
\mathrm{D}(\mathrm{LVNI})= & \mathrm{C}(2) *(\mathrm{LEX}(-1)-0.691538611086 * \mathrm{LOP}(-1)+16.4400376713 * \mathrm{LCPI}(-1)- \\
& 5.56253199491 * \mathrm{LM} 2(-1)-3.9138817517 * \mathrm{LIR}(-1))+\mathrm{C}(4) * \mathrm{D}(\mathrm{LVNI}(-1)) \\
& +\mathrm{C}(8) * \mathrm{D}(\mathrm{LCPI}(-1))
\end{aligned}
$$

\section{Bảng 7}

Kết quả ước lượng mô hình VECM

Coefficient

\begin{tabular}{ccccc}
\hline \hline $\mathrm{C}(1)$ & -0.062591 & 0.018495 & -3.384315 & 0.0009 \\
$\mathrm{C}(2)$ & -0.096356 & 0.047786 & -2.016394 & 0.0451 \\
$\mathrm{C}(3)$ & 0.075709 & 0.052123 & 1.452511 & 0.1479 \\
$\mathrm{C}(4)$ & 0.339593 & 0.064003 & 5.305881 & 0.0000 \\
$\mathrm{C}(5)$ & -0.000967 & 0.696960 & -0.001387 & 0.9989 \\
$\mathrm{C}(6)$ & 0.154119 & 0.175755 & 0.876895 & 0.3816 \\
$\mathrm{C}(7)$ & 0.038119 & 0.076170 & 0.500453 & 0.6173 \\
$\mathrm{C}(8)$ & -1.963948 & 0.938919 & -2.091712 & 0.0377 \\
$\mathrm{C}(9)$ & 0.471034 & 0.438888 & 1.073245 & 0.2844 \\
$\mathrm{C}(10)$ & 0.126245 & 0.112298 & 1.124196 & 0.2622 \\
\hline \hline
\end{tabular}

Nguồn: Kết quả ước lượng mô hình VECM trên Eview 10.0 


\subsection{Thảo luận kết quả nghiên cứu}

Từ kết quả nghiên cứu trên cho thấy:

Giá dầu (LOP) có tác động không đáng kể đến chỉ số VN-Index với hệ số hồi quy không có ý nghĩa thống kê. Điều này phù hợp với kết luận giá dầu không tác động đến chỉ số giá chứng khoán trong dài hạn và tác động tích cực trong ngắn hạn thể hiện trong nghiên cứu của Alqattan và Alhayky (2016).

Chỉ số giá tiêu dùng $(\mathrm{CPI})$ đại diện cho lạm phát có hệ số hồi quy bằng 4.642844 và có ý nghĩa thống kê cho thấy lạm phát có tác động cùng chiều đến chỉ số VN-Index trong dài hạn. Khi lạm phát tăng $1 \%$ với điều kiện các yếu tố khác không đổi sẽ làm chỉ số VN-Index tăng lên khoảng 4.64\%. Kết quả này phù hợp với nghiên cứu của Mousa và cộng sự (2012); Adam và Tweneboah (2008); ... Kết quả này hàm ý rằng mặc dù lạm phát tăng thể hiện sự bất ổn trong nền kinh tế, ảnh hưởng tiêu cực đến TTCK trong ngắn hạn, nhưng trong dài hạn, thị trường sẽ phân bổ hiệu quả các nguồn lực bằng cách điều chỉnh tăng theo mức giá tăng chung.

Lãi suất có tác động ngược chiều đến chỉ số VN-Index thể hiện qua hệ số hồi quy âm và có ý nghĩa. Kết quả nghiên cứu chỉ ra nếu lãi suất tăng $1 \%$ trong điều kiện các yếu tố khác không đổi sẽ làm chỉ số VN-Index giảm khoảng 1.8\%. Kết quả này phù hợp với các nghiên cứu thực nghiệm trước đó như Alam và Uddin (2009); Adam và Tweneboah (2008); ... Lãi suất tăng làm chi phí nguồn vốn doanh nghiệp tăng từ đó giảm lợi nhuận kỳ vọng, giá cổ phiếu giảm. Mặc dù vậy, trong ngắn hạn, lãi suất tăng làm tăng chỉ số giá chứng khoán. Điều này có thể là do TTCK chịu tác động mạnh mẽ hơn từ việc mở rộng cung tiền của NHNN và nhà đầu tư cần có thời gian để cân nhắc thay đổi danh mục đầu tư.

Cung tiền (M2) có hệ số hồi quy không có ý nghĩa thống kê nên cung tiền không ảnh hưởng đến chỉ số giá chứng khoán trong dài hạn. Bên cạnh đó, kết quả cũng cho thấy cung tiền tác động tích cực đến chỉ số giá chứng khoán trong ngắn hạn. Điều này phù hợp với lý thuyết kinh tế cho rằng khi ngân hàng trung ương tăng cung tiền sẽ làm giảm lãi suất, từ đó kích thích doanh nghiệp sản xuất, tăng đầu tư, phát triển thị trường. Tuy nhiên nếu cứ tiếp tục tăng như vậy sẽ làm cho nền kinh tế rơi vào tình trạng khủng hoảng thừa. Ngoài ra sự gia tăng tiền sẽ làm tăng giá cả của các yếu tố đầu vào, từ đó làm giảm tốc độ phát triển kinh tế cũng như TTCK.

Tỷ giá $(E X)$ không tác động đến chỉ số VN-Index trong dài hạn. Điều này đúng với kết quả nghiên cứu của Nguyen và Nguyen (2013). Bên cạnh đó, kết quả mô hình VECM cho kết quả tỷ giá tác động tiêu cực đến chỉ số VN-Index trong ngắn hạn. Khi tỷ giá tăng, sẽ mang đến cho các nhà đầu tư nhiều cơ hội kinh doanh ở thị trường ngoại hối hơn là đầu tư vào TTCK. Do đó, nhà đầu tư sẽ rút vốn để đầu tư vào ngoại tệ.

Giá vàng (GP) không có tác động đáng kể đến chỉ số VN-Index trong cả ngắn hạn và dài hạn. Mặc dù theo các nghiên cứu trước, giá vàng có tác động tiêu cực đến TTCK nhưng ở nước ta, vàng chưa phải là một kênh đầu tư lớn, mà vàng chủ yếu được coi là phương tiện cất trữ giá trị. Việc vàng tăng giá mạnh có thể tác động đến TTCK và làm cho tiền gửi trong dân 
cư giảm và như vậy sẽ ảnh hưởng giảm cung quỹ cho vay đối với nền kinh tế. Tuy nhiên, mức ảnh hưởng đó là không lớn Dinh và Nguyen (2008).

\section{Một số kiến nghị}

Kết quả nghiên cứu cho thấy các nhân tố vĩ mô có tác động đáng kể đến chỉ số giá chứng khoán. Do đó, các nhà làm chính sách cần quan tâm đến các chính sách tác động đến các nhân tố vĩ mô để gián tiếp phát triển TTCK nói riêng và nền kinh tế nói chung.

\subsection{Chính sách kiểm soát lạm phát}

Lạm phát là một trong những nguyên nhân gây bất ổn kinh tế, làm xói mòn niềm tin của nhà đầu tư. Mặc dù kết quả nghiên cứu cho thấy lạm phát có tác động tích cực đến chỉ số giá chứng khoán trong dài dạn, tuy nhiên trong ngắn hạn nó vẫn gây tác động tiêu cực gây ảnh hưởng tâm lý của nhà đầu tư. Vì vậy, trong điều hành kinh tế vĩ mô nhà hoạch định chính sách cần phải kiềm chế và kiểm soát lạm phát trong giới hạn hợp lý bằng các biện pháp thắt chặt cung tiền tệ và mở rộng cầu tiền tệ. Bên cạnh đó, kiểm soát chặt chẽ phương án giá và mức giá đối với các hàng hóa, dịch vụ do Nhà nước định giá; Giám sát chặt chẽ kê khai giá của doanh nghiệp đối với mặt hàng bình ổn, mặt hàng kê khai giá; ... Tất cả những mặt hàng, dịch vụ mà Nhà nước định giá thì cần điều chỉnh cho phù hợp, theo từng thời điểm, tránh hiện tượng tăng giá ồ ạt.

Tiếp tục hoàn thiện hệ thống pháp luật về giá, trong đó có việc hoàn thiện các định mức kinh tế - kỹ thuật làm cơ sở xác định giá dịch vụ theo lộ trình bảo đảm tính đúng, tính đủ chi phí thực hiện dịch vụ vào giá. Trường hợp mức giá cao hơn mức phí hiện hành thì cần có lộ trình điều hành phù hợp bảo đảm mục tiêu kiểm soát lạm phát. Ngoài ra, các bộ, ngành cần thực hiện tổ chức và quản lý tốt thị trường trong nước, ổn định tâm lý thị trường.

Mặt khác, một trong những nguyên nhân dẫn đến lạm phát là niềm tin của người dân vào các chính sách vĩ mô. Nếu người dân còn quan ngại về những bất ổn trong chính sách, luôn kỳ vọng vào sự mất giá của đồng tiền thì lạm phát cũng bị đẩy lên cao. Vì thế, việc khắc phục vấn đề này cần một sự nhất quán và minh bạch trong điều hành chính sách, tránh tạo ra các cú sốc, đồng thời thông tin kịp thời việc thay đổi chính sách đến người dân, chú ý đến định hướng dư luận xã hội, ngăn chặn kịp thời các tin đồn trên thị trường tiền tệ. Đây là những việc làm rất cần thiết để tạo niềm tin cho từng doanh nghiệp và cho các tầng lớp dân cư trong điều kiện hiện nay.

\subsection{Chính sách lãi suất}

Sự thay đổi của lãi suất sẽ ảnh hưởng đến nhu cầu đầu tư, xuất khẩu, ... Do đó ảnh hưởng đến tổng sản phẩm quốc dân. Đồng thời sự thay đổi lãi suất cũng ảnh hưởng đến nhu cầu tiêu dùng, ảnh hưởng đến cầu hàng hóa. Vì vậy, thông qua lãi suất, ngân hàng có thể thực hiện mục tiêu chính sách tiền tệ quốc gia.

Do lãi suất tác động tiêu cực đến chỉ số giá chứng khoán trong dài hạn, tác giả kiến nghị điều hành lãi suất ổn định lãi suất theo hướng giảm dần, qua đó góp phần hỗ trợ cung cấp vốn 
cho các hoạt động sản xuất kinh doanh của các chủ thể trong nền kinh tế góp phần hỗ trợ thúc đẩy tăng trưởng kinh tế vĩ mô, ổn định thị trường.

\subsection{Chính sách cung tiền}

Kết quả nghiên cứu cho thấy cung tiền có quan hệ cùng chiều với chỉ số giá chứng khoán, khi cung tiền tăng lên tác động làm chỉ số giá chứng khoán tăng theo. Vì vậy, ngân hàng trung ương cần điều hành chính sách tiền tệ một cách linh hoạt để kiểm soát cung tiền nhằm hỗ trợ thúc đẩy tăng trưởng kinh tế, qua đó gián tiếp phát triển TTCK. Ngân hàng trung ương cần có định hướng và lập kế hoạch điều chỉnh cung tiền trong nền kinh tế để đạt được mục tiêu đề ra. Cụ thể, cần điều hành linh hoạt, bám sát các diễn biến thị trường tài chính tiền tệ trong nước và quốc tế để đạt được mục tiêu kiểm soát lạm phát, ổn định thị trường ngoại tệ, góp phần ổn định kinh tế vĩ mô. Ngân hàng Nhà nước sẽ duy trì mặt bằng lãi suất hợp lý, phù hợp diễn biến kinh tế vĩ mô. Từng bước phấn đấu giảm hơn nữa lãi suất khi có điều kiện thích hợp. Không bơm tiền ra thị trường ồ ạt dẫn đến tình trạng không hấp thụ được. Từ góc độ ngân hàng, có thể hỗ trợ cho vay đối với các nhà đầu tư kinh doanh chứng khoán nhằm tăng nguồn vốn đầu tư để phát triển TTCK.

\subsection{Các chính sách khác}

Chỉ số VN-Index chịu tác động của giá dầu thế giới (Brent) và tỷ giá hối đoái chứng tỏ TTCK Việt Nam ngoài chịu ảnh hưởng của các yếu tố trong nước còn bị ảnh hưởng của yếu tố bên ngoài. Do đó, các nhà đầu tư cần phải quan tâm đến chính sách vĩ mô trong nước và cả những biến động bên ngoài.

Về phía chính phủ, trong việc điều hành chính sách tài khóa cần đẩy mạnh đầu tư công, hiệu quả của đồng vốn phải được đặt lên hàng đầu. Cần đưa ra lộ trình để giảm bội chi ngân sách, để có thể cân bằng được thu chi ngân sách.

\section{Tài liệu tham khảo}

Abdalla, I. S., \& Murinde, V. (1997). Exchange rate and stock price interactions in emerging financial markets: Evidence on India, Korea, Pakistan and the Philippines. Applied Financial Economics, 7(1), 25-35.

Adam, A. M., \& Tweneboah, G. (2008). Macroeconomic factors and stock market movement: Evidence from Ghana. Retrieved July 10, 2019, from https://mpra.ub.unimuenchen.de/13699/1/stock_market_and_macroeconomic_variables33.pdf

Akbar, M., Iqbal, F., \& Noor, F. (2019). Bayesian analysis of dynamic linkages among gold price, stock prices, exchange rate and interest rate in Pakistan. Resources Policy, 62, 154164. 
Alam, M., \& Uddin, G. (2009). Relationship between interest rate and stock price: Empirical evidence from developed and developing countries. International Journal of Business and Management, 4(3), 43-51.

Alqattan, A. A., \& Alhayky, A. (2016). Impact of oil prices on stock markets: Evidence from gulf cooperation council (GCC) financial markets. Amity Journal of Finance, 1(1), 1-8.

Bui, Y. K. (2013). Giáo trình thị trưòng chưng khoán [Stock market curriculum]. Hanoi, Vietnam: NXB Lao động-Xã hội.

Cecchetti, S. G., Schoenholtz, K. L., \& Fackler, J. (2006). Money, banking, and financial markets. New York, NY: McGraw-Hill/Irwin.

Devereux, M. B., \& Yetman, J. (2002). Menu costs and the long-run output-inflation trade-off. Economics Letters, 76(1), 95-100.

Dickey, D. A., \& Fuller, W. A. (1979). Distribution of the estimators for autoregressive time series with a unit root. Journal of the American Statistical Association, 74, 427-431.

Dinh, L. T. T., \& Nguyen, T. T. T. (2008). Tác động của tỷ giá, bất động sản, giá vàng lên thị trường chứng khoán Việt Nam thời gian qua [The impact of exchange rates, real estate, and gold prices on Vietnam's stock market recently]. Tạp chí Ngân hàng, 17, 26-30.

Friedman, M., \& Schwartz, A. J. (1965). Money and business cycles. In The state of monetary economics (pp. 32-78). Cambridge, MA: NBER.

Geetha, C., Mohidin, R., Chandran, V. V., \& Chong, V. (2011). The relationship between inflation and stock market: Evidence from Malaysia, United States and China. International Journal of Economics and Management Sciences, 1(2), 1-16.

González, M., Nave, J., \& Rubio, G. (2018). Macroeconomic determinants of stock market betas. Journal of Empirical Finance, 45, 26-44.

Hafer, R. W., \& Hein, S. E. (2007). The stock market. Westport, CO: Greenwood Publishing Group.

Hamilton, J. D. (2008). Understanding crude oil prices. Retrieved July 22, 2019, from https://www.nber.org/papers/w14492

Ho, L.-C., \& Huang, C.-H. (2015). The nonlinear relationships between stock indexes and exchange rates. Japan and the World Economy, 33, 20-27.

Le, H. T. T., \& Dang, D. V. (2017). Giáo trình lý thuyết tài chính tiền tệ [Textbook of financial and monetary theory]. Ho Chi Minh, Vietnam: NXB Kinh tế.

Malkiel, B. G., \& Fama, E. F. (1970). Efficient capital markets: A review of theory and empirical work. The Journal of Finance, 25(2), 383-417. 
Maysami, R. C., \& Koh, T. S. (2000). A vector error correction model of the Singapore stock market. International Review of Economics \& Finance, 9(1), 79-96.

Mokni, K., \& Youssef, M. (2019). Measuring persistence of dependence between crude oil prices and GCC stock markets: A copula approach. The Quarterly Review of Economics and Finance, 72, 14-33.

Mousa, S. N., Safi, W. A., Hasoneh, A., \& Mohammad, M. (2012). The relationship between inflation and stock prices: A case of Jordan. International Journal of Applied Agricultural Research, 10, 46-52.

Nguyen, K. M., \& Nguyen, D. V. (2013). Quan hệ giữa các yếu tố kinh tế vĩ mô và biến động thị trường chứng khoán: Bằng chứng nghiên cứu từ thị trường Việt Nam [Relationship between macroeconomic factors and stock market volatility: Research evidence from Vietnam market]. Tạp chí Phát triển Khoa học và Công nghệ, 16(3Q), 86-100.

Phillips, P. C. B., \& Perron, P. (1988). Testing for unit roots in time series regression. Biometrika, 75, 335-346.

Rahman, A. A., Sidek, N. Z. M., \& Tafri, F. H. (2009). Macroeconomic determinants of Malaysian stock market. African Journal of Business Management, 3(3), 95-106.

Rjoub, H. (2012). Stock prices and exchange rates dynamics: Evidence from emerging markets. African Journal of Business Management, 6(13), 4728.

Rozeff, M. S. (1974). Money and stock prices: Market efficiency and the lag in effect of monetary policy. Journal of Financial Economics, 1(3), 245-302.

Schwert, G. W. (1989). Why does stock market volatility change over time? The Journal of Finance, 44(5), 1115-1153.

Singhal, S., Choudhary, S., \& Biswal, P. C. (2019). Return and volatility linkages among international crude oil price, gold price, exchange rate and stock markets: Evidence from Mexico. Resources Policy, 60, 255-261.

Smyth, R., \& Narayan, P. K. (2018). What do we know about oil prices and stock returns? International Review of Financial Analysis, 57, 148-156.

Su, Z., Fang, T., \& Yin, L. (2019). Understanding stock market volatility: What is the role of U.S. uncertainty? The North American Journal of Economics and Finance, 48, 582-590.

Subhani, M. I., Gul, A., \& Osman, A. (2010). Relationship between consumer price index (CPI) and KSE-100 index trading volume in pakistan and finding the endogeneity in the involved data. Retrieved July 21, 2019, from https://www.researchgate.net/publication/47760183_Relationship_between_consumer_ price_index_CPI_and_KSE-

100_index_trading_volume_in_pakistan_and_finding_the_endogeneity_in_the_involve d_data 
Tran, H. H. (2017). Tác động của giá dầu thế giới đến thị trương chứng khoán và các biến vĩ mô của nền kinh tế: Trương hợp Việt Nam [The impact of world oil prices on the stock market and macroeconomic variables: The case of Vietnam]. Retrieved July 20, 2019, from Tạp chí Công thương website: http://tapchicongthuong.vn/bai-viet/tac-dong-cuagia-dau-the-gioi-den-thi-truong-chung-khoan-va-cac-bien-vi-mo-trong-nen-kinh-tetruong-hop-viet-nam-51084.htm

Truong, L. D. (2014). Các nhân tố ảnh hưởng đến sự thay đổi giá của cổ phiếu: Các bằng chứng từ Sở Giao dịch Chứng khoán Thành Phố Hồ Chí Minh [Factors influencing stock price changes: Evidence from Ho Chi Minh City Stock Exchange]. Tạp chi Khoa hoc Truòng Đại học Cần Tho, 72-78.

Wei, Y., Qin, S., Li, X., Zhu, S., \& Wei, G. (2019). Oil price fluctuation, stock market and macroeconomic fundamentals: Evidence from China before and after the financial crisis. Finance Research Letters, 30, 23-29. 